\title{
Nuclear Structure and Radioactive Decay Resources at the US National Nuclear Data Center
}

\author{
A.A. Sonzogni, T.W. Burrows, B. Pritychenko, J.K. Tuli, and D.F. Winchell \\ National Nuclear Data Center, Building 197D, Brookhaven National Laboratory, Upton, NY 11973-5000, USA
}

Abstract. The National Nuclear Data Center has a long tradition of evaluating nuclear structure and decay data as well as offering tools to assist in nuclear science research and applications. With these tools, users can obtain recommended values for nuclear structure and radioactive decay observables as well as links to the relevant articles. Some of the activities are described in this article.

\section{ENSDF}

The Evaluated Nuclear Structure Data File (ENSDF) stores recommended nuclear structure and decay data for all nuclei. ENSDF deals with properties such as:

- nuclear level energies, spin and parity, half-life and decay modes,

- nuclear radiation energy and intensity for different types,

- nuclear decay modes and their probabilities.

The data in ENSDF is incorporated after a careful compilation and analysis followed by a peer-reviewed refereeing process. Nearly 40 physicists worldwide contribute to ENSDF. In the last 4 years, an average of 230 nuclides was evaluated per year and nearly $80 \%$ of the evaluation work was performed by evaluators from the USA and Canada. The management of ENSDF resides at the NNDC, which is also responsible for the maintenance of the many codes used in the evaluations.

The contents of ENSDF are stored in a relational database, which can be accessed electronically at http://www.nndc.bnl.gov/ensdf. This web site contains several search forms as well as an index to the database. ENSDF evaluations for $\mathrm{A}=3-20$ are published in Nuclear Physics A, the rest are published in the Nuclear Data Sheets journal, published by Elsevier, http://www.nndc.bnl.gov/nds. More details about ENSDF can be found in earlier publications [1].

Any nuclide that has been reported as experimentally observed is included in ENSDF. As of today, it includes 3,011 nuclides with a total of 146, 314 nuclear levels. In order to illustrate the richness of the database and our current knowledge of nuclear structure, two plots are presented. In figure 1 we plot the distribution of largest spin values. At the extreme of the distribution is ${ }^{152}$ Dy with a $\mathrm{J}^{+}=68^{+}$excited level, with other rare earth nuclides also showing very high angular momentum values. One can also query the database to plot the number of levels distribution, which is shown in figure 2. In this case, the nuclei at the extreme are two doubly magic cases, ${ }^{40} \mathrm{Ca}$ and ${ }^{208} \mathrm{~Pb}$, both with more than 500 levels.

\section{NSR}

NSR (Nuclear Science References) is a bibliographic database containing nearly 200,000 nuclear science articles, indexed

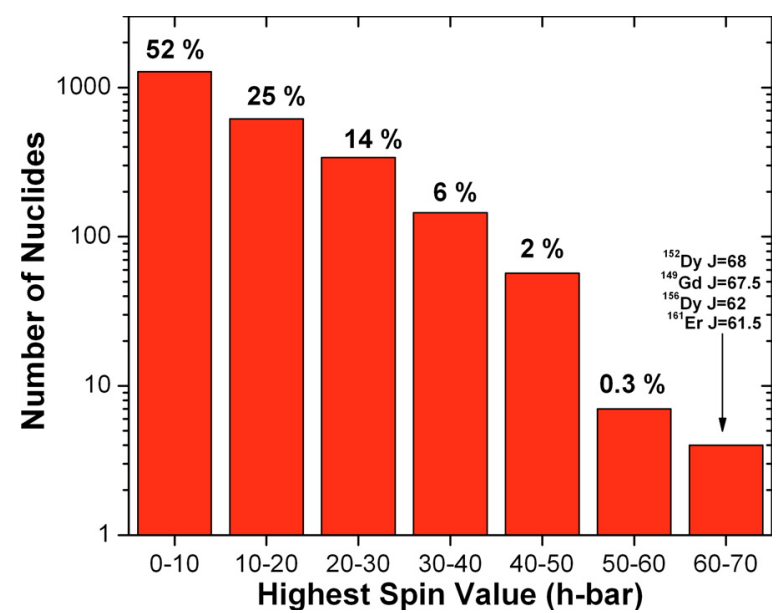

Fig. 1. Maximum angular momentum distribution for all nuclides in ENSDF, about $52 \%$ of them have maximum spin values below 10 h-bar units.

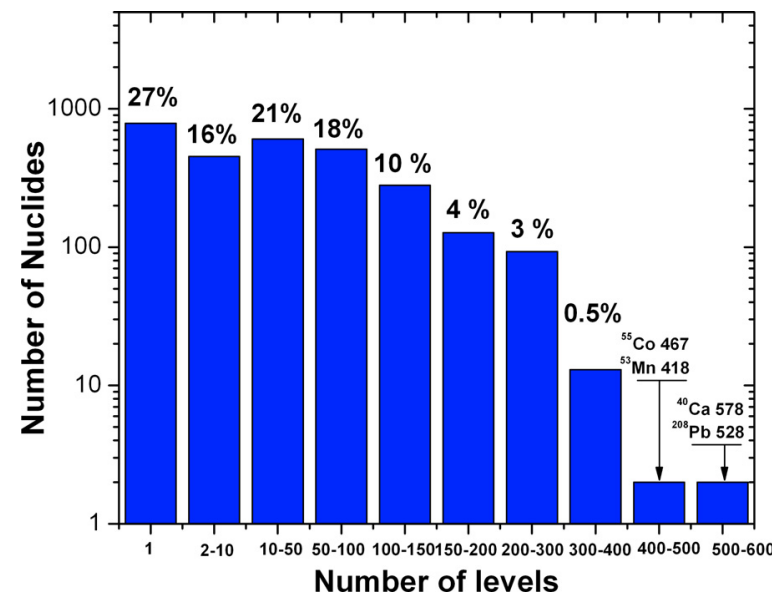

Fig. 2. Number of levels distribution for all nuclides in ENSDF. For about $27 \%$ of them, only 1 level is known, while ${ }^{48} \mathrm{Ca}$, with 578 levels, has the largest number.

according to content. About 4,000 are added each year covering 80 journals as well as conference proceedings and laboratory reports. Each article included in NSR is read assigned a number of variables that succinctly described the 


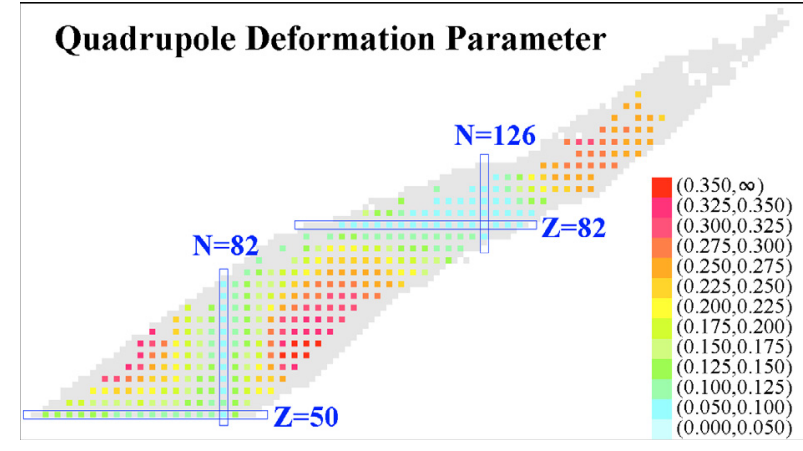

Fig. 3. Quadrupole deformation obtained from Grodzin's formula for even-even nuclides with $\mathrm{Z}$ above 50, using data retrieved from $\mathrm{NuDat}$ (http://www.nndc.bnl.gov/nudat2/). Neutron and proton magic numbers are marked.

article. These variables, also known as keywords, are then incorporated in a database.

One unique feature of NSR is the ability to selectively retrieve articles out of a vast number, satisfying a particular set of conditions. A classical example is to search for articles reporting new measurements of the ${ }^{137} \mathrm{Cs}$ half-life in the last 4 years. Many search products will give a list of articles containing the words "137Cs" and "half-life" regardless of their correlation. NSR, on the other hand, will give the right answer. More details on NSR and its deep interplay with ENSDF evaluations can be found in [2].

\section{NuDat}

NuDat is a software product with two main goals, to present nuclear structure and decay information from ENSDF in a user-friendly way, and to allow users to execute complex search operations in the wealth of data contained in ENSDF. $\mathrm{NuDat}$ provides an interactive chart of nuclides for navigation and an output in the Table of Isotopes style. NuDat contents are updated regularly as new evaluations are entered into ENSDF.

Among the many search possibilities in NuDat, the triple gamma coincidence query is the most CPU intensive, which allows to search for the nucleus that has 2 or 3 gamma rays in coincidence. Figure 3 exemplifies another search case, where the first $2^{+}$level energy for even-even nuclides was looked for as a function of $\mathrm{Z}$ and $\mathrm{A}$ for $\mathrm{Z}$ larger than or equal to $50(\mathrm{Sn})$, and then, the quadrupole deformation parameter was deduced using Grodzin's formula. Experimentally known nuclides with no quadrupole deformation data are plotted as a grey cell. The high-deformation regions at mid-shell are clearly seen.

\section{ENDF}

The recently released ENDF/B-VII.0 library [3] contains a decay data sub-library which has been derived mostly from ENSDF and the Nuclear Wallet Cards. The ENDF-6 format in which this library was written has the advantage that the decay data can be easily used in complex network calculations. An example of this can be seen in figure 4, which shows

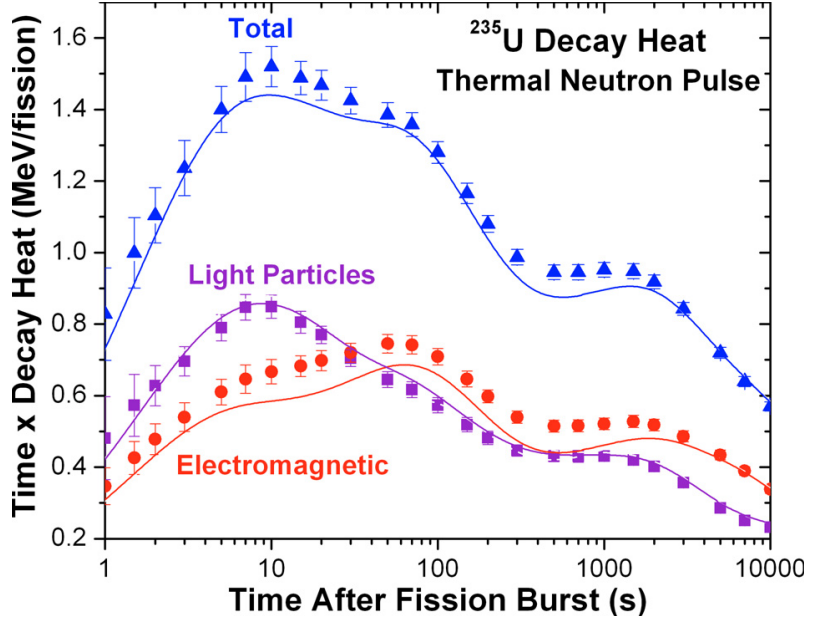

Fig. 4. Decay heat as function of time after fission, for a thermal neutron pulse on ${ }^{235} \mathrm{U}$. The full lines are obtained following decay network calculation using the ENDF/B-VII.0 decay data sub-library.

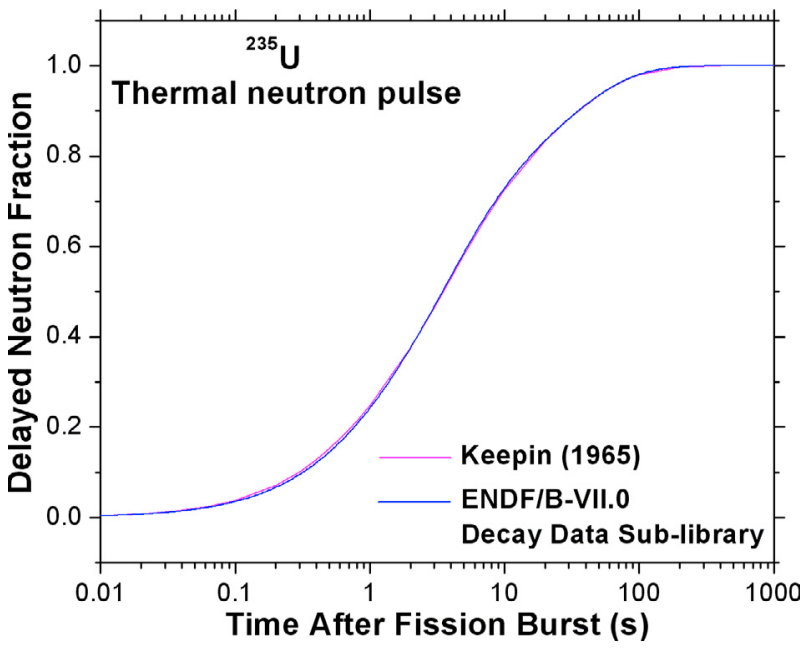

Fig. 5. Delayed neutron fraction as function of time after fission, for a thermal neutron pulse on ${ }^{235} \mathrm{U}$. The 1965 Keepin parameterisation is compared with a decay network calculation using the ENDF/BVII.0 decay data sub-library.

the decay heat as function of time following a neutroninduced fission burst in ${ }^{235} \mathrm{U}$. Another example is shown in figure 5 where the delayed neutron fraction is plotted as a function of time following a thermal fission event. The result obtained using the decay data sub-library in a decay network calculation is compared with the Keepin parameterisation [4]. The contents of this sub-library as well as any part of the ENDF/B-VII.0 library and other major regional libraries of this type (JEFF-3.1, JENDL-3.3 and ENDF/B-VI.8) can be downloaded from the recently developed Sigma interface, http://www.nndc.bnl.gov/sigma.

\section{Other tools}

Several other tools of use in Nuclear Structure research are offered online.

- Q-values (www.nndc.bnl.gov/qcalc): Nuclear reaction and decay Q-values can be calculated using the QCalc tool, 
which uses the 2003 atomic mass evaluation of Audi et al. [5].

- B(E2) values (www.nndc.bnl.gov/be2): A database and corresponding webpage have been recently set up containing reduced transition probabilities for $0^{+}$to $2^{+}$transitions in even-even nuclei. The data are based on the evaluation work for Raman et al. [6].

- Double beta decay (www.nndc.bnl.gov/bbdecay): An evaluation of experimental double-beta decay half-lives has recently been completed.

\section{Summary}

In summary, we have presented short descriptions on ENSDF, NSR, NuDat, the new ENDF decay data library and some nuclear structure tools. The way these data is offered to the public has undergone a drastic improvement in the last few years due to advancements in information technology. We can expect further changes as the format used to store these data is modernized.
This work was sponsored by the Office of Nuclear Physics, Office of Science, US Department of Energy, under contract DE-AC0298CH10886 with Brookhaven Science Associates, LLC.

\section{References}

1. J.K. Tuli, in Proceedings of the International Conference on Nuclear Data for Science and Technology, 2004, edited by R.C. Haight, M.B. Chadwick, T. Kawano, P. Talou, AIP Conference Proceedings 769 (2005), p. 265.

2. D.F. Winchell, in Proceedings of the International Conference on Nuclear Data for Science and Technology, 2004, edited by R.C. Haight, M.B. Chadwick, T. Kawano, P. Talou, AIP Conference Proceedings 769 (2005), p. 570. C.M. Baglin, ibid. p. 231.

3. M.B. Chadwick et al., Nucl. Data Sheets 107, 2931 (2006).

4. G.R. Keepin, Physics of Neutron Kinetics (Addison-Wesley, New York, 1965).

5. G.Audi, A.H. Wapstra, C. Thibault, Nucl. Phys. A 729, 337 (2003).

6. S. Raman, C.W. Nestor, P. Tikkanen, At. Data Nucl. Data Tables 78, 1 (2001). 